Check for updates

Cite this: RSC Adv., 2017, 7, 36555

Received 16th May 2017

Accepted 19th July 2017

DOI: $10.1039 / c 7 r a 05510 b$

rsc.li/rsc-advances

\section{Insights into iron induced fouling of ion-exchange membranes revealed by a quartz crystal microbalance with dissipation monitoring $\dagger$}

\begin{abstract}
Mei Chen, ${ }^{a}$ Jinxing Ma, (D) ${ }^{* b}$ Zhiwei Wang, ${ }^{a}$ Xingran Zhang ${ }^{a}$ and Zhichao Wu ${ }^{a}$
Understanding the mechanisms of multivalent iron interacting with ion-exchange membranes (IEMs) is crucial for the prediction of membrane fouling as well as the development of control strategies. In this study, the adsorption and desorption behaviors of Fe(III) species on a typical IEM, Nafion, were investigated using a quartz crystal microbalance with dissipation monitoring (QCM-D). The Nafion thin film formed on the crystal sensor surface via a sedimentation method showed a non-rigid structure with a Voigt-based mass concentration of $\sim 500 \mathrm{ng} \mathrm{cm}^{-2}$ at a Nafion solution injection time of $10 \mathrm{~min}$. Adsorption of $\mathrm{Fe}(\mathrm{II})$ species was first assessed using a $10 \mathrm{mM} \mathrm{Fe}(\mathrm{II})$ solution, followed by rinsing under different conditions to induce the structural transformation and/or release of Fe(III) from the Nafion film. The QCM-D results suggested that there was a rapid deposition of Fe(III) at the initial stage. It has been found that the ongoing adsorption process exhibiting pseudo-first-order kinetics was associated with the interaction between $\mathrm{Fe}(\mathrm{II})$ and the surface functional sites $\left(-\mathrm{SO}_{3} \mathrm{H}\right)$ of Nafion, which consequently retarded the proton transfer. Compared to the rinse with an acidic solution $(\mathrm{HCl}$ in ultrapure water, $\mathrm{pH}$ of 2.37), the QCM-D results of neutral elution (ultrapure water, $\mathrm{pH}$ of 6.50 ) indicated the hydrolysis of $\mathrm{Fe}(\mathrm{III})$ and/or structural transformation of the $\mu$-oxo bridged, $\mathrm{Fe}-\mathrm{O}-\mathrm{Fe}$.
\end{abstract}

\section{Introduction}

Ion-exchange membranes (IEMs) are insoluble matrices generally made from polymeric materials attached to charged ion groups with a preference to conduct dissolved cations or anions (e.g., $\mathrm{Na}^{+}$and $\mathrm{Cl}^{-}$) whilst being much less permeable to gases and electrolytes. ${ }^{1}$ The conventional applications of IEMs lie in the use of an electrical potential or concentration gradient to selectively transport cations or anions. For example, when applied in chlor-alkali production, IEMs are typically arranged between the electrodes with a galvanic potential provided as a voltage generated at the electrodes. An emerging interest in IEMs is the incorporation into water/wastewater treatment technologies with more recent innovations including microbial fuel cells (MFCs), microbial desalination cells (MDCs), capacitive deionization (CDI) and capacitive mixing (CapMix). ${ }^{2-6}$

Despite the prominent progress in environmental applications of IEMs, crucial concerns remain with regard to (i) the non-target ion transfer and (ii) fouling that leads to the

${ }^{a}$ State Key Laboratory of Pollution Control and Resources Reuse, College of Environmental Science and Engineering, Tongji University, Shanghai, 200092, PR China

${ }^{b}$ School of Civil and Environmental Engineering, University of New South Wales, Sydney, NSW 2052, Australia. E-mail: jinxing.ma@unsw.edu.au; Tel: +61293859501 $\dagger$ Electronic supplementary information (ESI) available. See DOI: 10.1039/c7ra05510b deterioration of the membrane performance. ${ }^{7-9}$ For instance, it has been well recognized that the metal cations are of great abundance in the substrates of MFCs and MDCs, which can transfer across the IEMs as non-target ions though some IEM types possess a preferential conductivity for protons such as 3.4 for $\mathrm{H}^{+} / \mathrm{Na}^{+}$and 6.2 for $\mathrm{H}^{+} / \mathrm{K}^{+}$in Nafion $117 .{ }^{10}$ The influences of non-proton transfer are profound as this would result in (i) $\mathrm{pH}$ excursion inhibiting the biocatalysts activity and (ii) the loss of thermodynamic cell potential. ${ }^{\mathbf{7 , 8 1 1}}$ Moreover, it has been reported that the movement of multivalent cations and/or their polynuclear species across the IEMs can further cause strong interaction with the charged groups (e.g., sulfonic acid groups $\left.\left(-\mathrm{SO}_{3} \mathrm{H}\right)\right),{ }^{7,9}$ which retards the "hop" of subsequent ions from one site to the adjacent sites and consequently increases the internal resistance. ${ }^{\mathbf{1 2}}$

Iron (Fe) is an important element involved in metabolism and redox chemistry. In surface and ground waters, Fe presents at varying concentration levels, ranging from 0.5 to $50 \mathrm{mg} \mathrm{L}^{-1} \cdot{ }^{13}$ In the anode chambers of MFCs and MDCs, the origin of aqueous $\mathrm{Fe}(\mathrm{II})$ and $\mathrm{Fe}(\mathrm{III})$ can be associated with the reductive dissolution of Fe-(oxyhydr)oxides in addition to the authigenic dissolved species. Moreover, $\mathrm{Fe}(\mathrm{II}) / \mathrm{Fe}(\mathrm{III})$ can be directly employed as an electron mediator. ${ }^{\mathbf{1 4}}$ When using IEMs in water/ wastewater treatment, considerations should be given to the interfacial behaviors of Fe species on IEMs since recent evidence has indicated that $\mathrm{Fe}(\mathrm{III})$ species are related with the mineral precipitation on IEMs with their further interaction 
with macromolecules resulting in biofouling that deteriorates the process performance. ${ }^{\mathbf{8} 9}$ Although previous studies using K-edge extended X-ray absorption fine structure (EXAFS) spectroscopy have implied that an oxo-bridged dimer, Fe-O-Fe, dominates the $\mathrm{Fe}$ speciation of the $\mathrm{Fe}(\mathrm{III})$ neutralized membranes (Nafion 125 IEM) followed by a structural transformation to edge-sharing $\mathrm{FeO}_{6}$ octahedra, ${ }^{15,16}$ there are still many characteristics of this process that remain unclear. For example, (i) what kind of behavior is prevailing during the adsorption and/or precipitation of Fe(III) on IEMs? (ii) How is this process influenced by the environmental factors (e.g., $\mathrm{pH})$ ? (iii) Will this lead to significant impacts on the performance of IEMs?

In this study, we have investigated the nature of iron induced fouling of a commercial IEM, Nafion 117, by using a quartz crystal microbalance with dissipation (QCM-D). QCM-D has been suggested as a useful method to obtain information on adherence and fouling propensity of foulants on membrane surfaces. ${ }^{17-19}$ Coating of Nafion film on gold-plated crystal sensors was conducted via a sedimentation method. The overarching goals of this work are, therefore, to (i) form Nafion thin film on crystal sensor surface, (ii) elucidate the behaviors of $\mathrm{Fe}(\mathrm{III})$ on the resultant film and (ii) evaluate the impacts of $\mathrm{Fe}$ precipitation on the membrane properties. An adequate understanding of the Fe induced fouling mechanisms of IEMs is expected to be conducive to advancing control strategies for IEM-related processes.

\section{Materials and methods}

\subsection{Reagents}

All chemicals were analytical reagent grade and used as received unless otherwise stated. All solutions were prepared using $18.2 \mathrm{M} \Omega \mathrm{cm}$ Milli-Q water (Millipore). Glassware was soaked in a $5 \% \mathrm{v} / \mathrm{v} \mathrm{HCl}$ for at least three days and rinsed prior to use. Nafion® 117 membrane (Product no. 274674) and perfluorinated resin solution (Nafion solution, Product no. 274704) were purchased from Sigma-Aldrich. $1 \mathrm{M} \mathrm{NaOH}$ and $1 \mathrm{M} \mathrm{HCl}$ were used for the adjustment of solution pH when necessary. Stock solution of $10 \mathrm{mM}$ ferric chloride hexahydrate $\left(\mathrm{FeCl}_{3}\right.$ $\cdot 6 \mathrm{H}_{2} \mathrm{O}$ ) was prepared for the following experiments, and according to the calculation using an equilibrium speciation program Visual Minteq, dominant $\mathrm{Fe}(\mathrm{III})$ species are $\mathrm{Fe}^{3+}$ (39.2\%), $\mathrm{FeOH}^{2+}(32.9 \%), \mathrm{Fe}_{2}(\mathrm{OH})_{2}{ }^{4+}(14.7 \%)$ and $\mathrm{FeCl}^{2+}$ $(10.5 \%)$ at the equilibrium $\mathrm{pH}$ of 2.37 .

\subsection{Quartz crystal microbalance with dissipation monitoring}

In this study, a quartz crystal microbalance with dissipation monitoring (QCM-D, E4, Q-Sense, Västra Frölunda, Sweden) system was used to monitor the sedimentation of Nafion on the crystal sensor surface and to investigate Fe(III) induced fouling of Nafion. The E4 system has four modules that allow a parallel configuration, and each module holds a $5 \mathrm{MHz}$ AT-cut quartz crystal sensor. Gold-plated crystal sensors (Q-Sense) were utilized for the following studies. Prior to use, the crystal sensors were subject to the cleaning protocols documented by the QCM-D manufacturer, with the flow modules cleaned with a $2.0 \%$ sodium dodecyl sulfate (SDS) solution followed by rinse with copious amount of ultrapure water. Prepared solutions were degassed through ultrasonication for $10 \mathrm{~min}$ and all experiments were performed at a constant flow rate of $150 \mu \mathrm{L}$ $\min ^{-1}$ using a peristaltic pump (IsmaTec, IDEX). Solution temperature inside the chamber was maintained at about $25^{\circ} \mathrm{C}$.

\subsection{Sedimentation of Nafion on QCM-D crystal sensors}

At the initial stage of the experiment, the frequency and dissipation responses of the crystal sensors at $n$ overtone $(n=3,5,7$, 9 and 11) were monitored whilst the system was rinsed with ultrapure water $(\mathrm{pH}=6.50)$ for stabilization (step I in Fig. 1). Stable baseline was considered to be achieved when the drift of normalized frequency signals at the $5^{\text {th }}$ overtone (i.e., $\Delta f_{5}$ ), which was chosen based on the best signal-to-noise ratio, was decreased below $0.5 \mathrm{~Hz}$ in a period of $10 \mathrm{~min}$. Subsequently the Nafion solution was introduced into the modules for the sedimentation of Nafion on crystal sensors (step II in Fig. 1), resulting in the decrease in the frequency (i.e., an increase of $|\Delta f|)$ due to the change of solution properties and/or adsorption of Nafion on the surface of crystal sensors. The sensors were then rinsed with ultrapure water (step III in Fig. 1) until stable frequency and dissipation signals of the $5^{\text {th }}$ overtone were achieved.

The Voigt-based model ${ }^{\mathbf{2 0 , 2 1}}$ was introduced herein to quantify the mass concentrations of Nafion on the QCM-D crystal surfaces. Briefly, the normalized frequency shifts $(\Delta f)$ and dissipation shifts $(\Delta D)$ at the $3^{\text {rd }}, 5^{\text {th }}, 7^{\text {th }}, 9^{\text {th }}$ and $11^{\text {th }}$ overtone collected from the experiments were used for model fitting using the Q-Tools 3.0 (Q-Sense), obtaining the surface concentration (mass and thickness), viscosity and shear modulus of the Nafion film as a function of time. The fluid density and viscosity of the Nafion solution were fixed at $0.87 \times 10^{3} \mathrm{~kg} \mathrm{~m}^{-3}$ and $6.0 \mathrm{mPa} \mathrm{s}$ according to the manufacturer, ${ }^{22}$ and those parameters for other solutions including ultrapure water and electrolyte were fixed at $1.00 \times 10^{3} \mathrm{~kg} \mathrm{~m}^{-3}$ and $1.0 \mathrm{mPa} \mathrm{s}$, respectively. According to a previous work on water-sorption properties of Nafion $117 \mathrm{H}^{23}$ the density of Nafion layers was about $1.10 \times 10^{3} \mathrm{~kg} \mathrm{~m}^{-3}$, which was employed for the Voigtbased model fitting in this study.

\subsection{Evaluation of adsorption of $\mathrm{Fe}(\mathrm{III})$}

Following the assembly and stabilization of the Nafion film, a $10 \mathrm{mM} \mathrm{Fe(III)} \mathrm{solution} \mathrm{was} \mathrm{injected} \mathrm{into} \mathrm{the} \mathrm{sensor} \mathrm{modules}$ for $20 \mathrm{~min}$ (step IV in Fig. 1), with control tests carried out under the same conditions but using pristine gold-plated crystal sensors. These sensors were then subject to rinse with ultrapure water ( $\mathrm{pH}$ of 6.50$)$ or acid solution $(\mathrm{HCl}$ in ultrapure water, $\mathrm{pH}$ of 2.37), respectively (step V in Fig. 1). The normalized frequency signals of the Nafion-coated crystals were obtained by deducting the initial $\Delta f$ of Nafion film. All experiments were conducted in triplicate with average value reported.

The relationship between the QCM-D results and Nafion membrane performance was further evaluated by measuring 


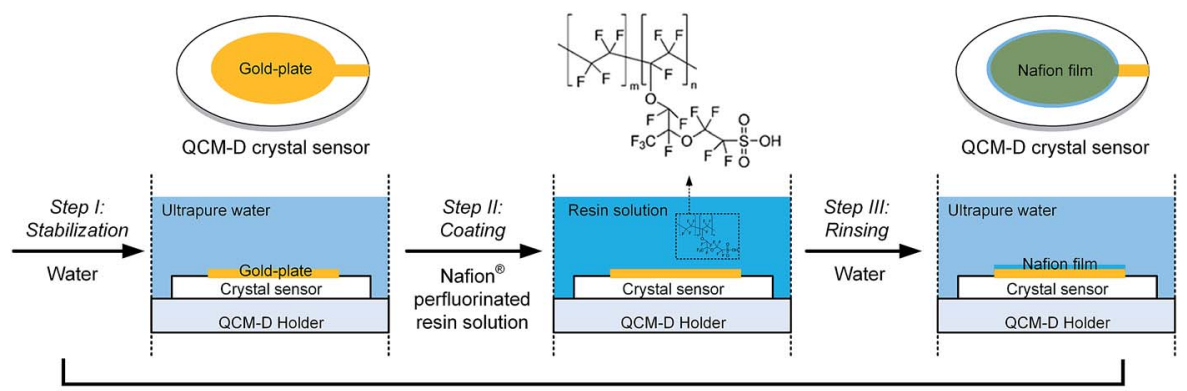

Sedimentation (assembly) of Nafion on crystal sensors

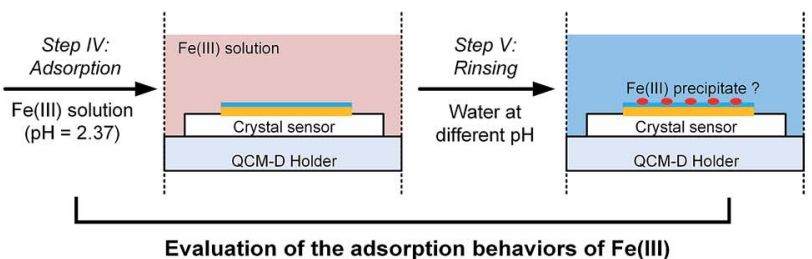

Fig. 1 Experimental workflow for the self-assembly of Nafion film on QCM-D crystal sensors and evaluation of the interfacial behavior of Fe(III) species.

the increase in membrane ohmic resistance as a result of $\mathrm{Fe}(\mathrm{III})$ fouling. An IEM of the same material, Nafion ${ }^{\circledR} 117$ membrane, was used to evaluate the consequent membrane performance after fouling. Briefly, $2 \mathrm{~cm} \times 2 \mathrm{~cm}$ Nafion coupons were soaked in the vessels containing $100 \mathrm{~mL}$ of $10 \mathrm{mM} \mathrm{Fe}$ (III) solution on an orbital shaker table at $150 \mathrm{rpm}$. Membranes were withdrawn at pre-determined time intervals. To evaluate the impacts of $\mathrm{Fe}$ (III) hydrolysis and/or structural transformation on Nafion performance, parallel experiments were conducted according to the procedure described above, except for the rinse of the withdrawn coupons with copious amount of ultrapure water $(\mathrm{pH}$ of 6.50). Ohmic resistances of the membranes $\left(R_{\mathrm{m}}\right)$ were measured according to a protocol outlined previously. ${ }^{9} 10 \mathrm{mM}$ Fe(III) was added into the working electrolyte to avoid the possible desorption of $\mathrm{Fe}(\mathrm{III})$ from the IEMs during the measurement in $0.1 \mathrm{M} \mathrm{KCl}$ solution.

\subsection{Other analytical methods}

The equivalent weight (EW) of the Nafion-H type materials was determined to be $1100 \pm 21$ by back titration of the hydrogen ion that was exchanged from Nafion with $2 \mathrm{M} \mathrm{NaOH}$ solution over 24 hours at ambient temperature $\left(\sim 25{ }^{\circ} \mathrm{C}\right)$. Ion exchange capacity (IEC) was calculated as the reciprocal of EW, i.e., IEC $=$ 1/EW. ${ }^{7}$ To evaluate the relationship between the loss of IEC and aqueous $\mathrm{pH}$, pre-weighed Nafion 1100EW membranes were first soaked in ultrapure water that was added with $1 \mathrm{M} \mathrm{HCl}$ or $1 \mathrm{M}$ $\mathrm{NaOH}$ solution to obtain a series of initial $\mathrm{pH}$ from 2.83 to 11.41. After either 2 or $24 \mathrm{~h}$ of reaction, membranes were gently rinsed with copious amount of ultrapure water and then IEC were measured according to the protocol described above.

Functional groups of the pristine and fouled Nafion membranes were analysed on an attenuated total reflectance Fourier transform infrared (ATR-FTIR) spectroscopy. The ATRFTIR spectra were collected using a Nicolet 5700 spectrometer
(Thermo Electron Co., U.S.) at a $4 \mathrm{~cm}^{-1}$ resolution in the range of 400 to $4000 \mathrm{~cm}^{-1}$. Prior to the measurement, the surface of the membranes were dried with the use of lens wiping paper following withdrawal from the solution. The membrane topography was also characterized by the atomic force microscope (AFM) technology (Multimode IV, Bruker Nano Surface, USA) with the Nanoscope ${ }^{\circledR}$ control software used for image acquisition.

\section{Results and discussion}

\subsection{Characteristics of the Nafion film}

Fig. 2a shows the time course results of $\Delta f_{5}$ and $\Delta D_{5}$ during the sedimentation (assembly) of Nafion films on the gold-plated crystal sensors. Injection of the Nafion solution led to
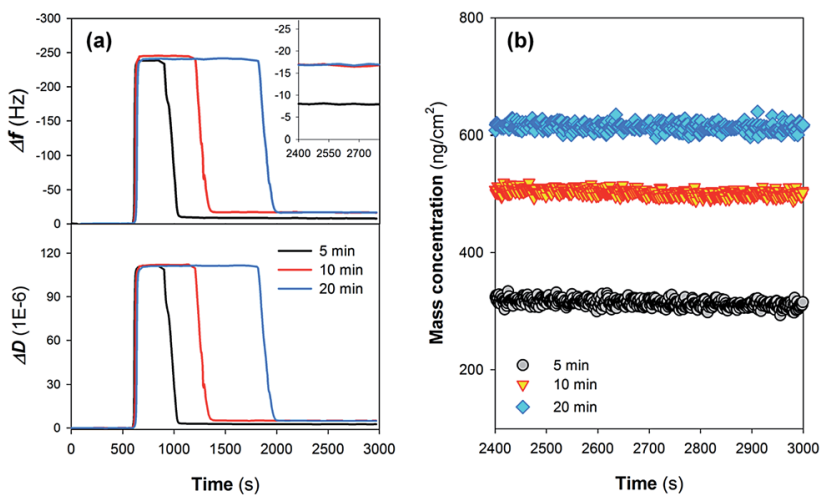

Fig. 2 Time course results of (a) representative frequency shift $\left(\Delta f_{5}\right)$ and dissipation shift $\left(\Delta D_{5}\right)$ describing the assembly of Nafion films on crystal sensors. Residual $\Delta f_{5}$ of the films following rinse with ultrapure water (step III in Fig. 1) are specifically shown in the inset. (b) Mass concentrations of the Nafion films as a function of Nafion injection time. 
a decrease in $\Delta f_{5}$ (i.e., an increase in $\left|\Delta f_{5}\right|$ ) (step II in Fig. 1) as a result of (i) the change of the properties of the fluid and/or (ii) the deposition of Nafion. It can be observed that the steady state $\Delta f_{5}$ following the injection of the Nafion solution was not increased with prolonging the injection time, suggesting that a dynamic adsorption balance could be established in a relatively short injection time. ${ }^{20}$ The subsequent rinse with ultrapure water (step III in Fig. 1) significantly decreased the resonance frequencies (i.e., a dramatic decrease in $\left|\Delta f_{5}\right|$ ). As can be seen from Fig. 2a, it is likely that a longer injection time for step II (Fig. 1) is in favor of the interaction between the Nafion and crystal surface: i.e., following the rinse, the residual $\Delta f_{5}(-16.7$ $\mathrm{Hz}$ ) at an injection time of $10 \mathrm{~min}$ is similar with that of $20 \mathrm{~min}$ $(-17.0 \mathrm{~Hz})$ whilst exhibiting a much higher absolute value than that of $5 \mathrm{~min}(-7.9 \mathrm{~Hz})$ (the inset figure of Fig. 2a). The timeresolved behavior of $\Delta D_{5}$ was comparable with that of $\Delta f_{5}$; after the rinse with ultrapure water for $1000 \mathrm{~s}$, the crystal sensors showed residual $\Delta D_{5}$ of $2.6,5.0$ and $4.9 \times 10^{-6}$ at an initial injection of Nafion solution for 5, 10 and $20 \mathrm{~min}$, respectively.

After stabilization of the residual response signals (following step III in Fig. 1), $\Delta f$ and $\Delta D$ at the $3^{\text {rd }}, 5^{\text {th }}, 7^{\text {th }}, 9^{\text {th }}$ and $11^{\text {th }}$ overtone were collected from the experiments and utilized for model fitting. Despite the injection of Nafion solution for 10 and 20 min resulting in similar residual $\Delta f_{5}$ (Fig. 2a), the Voigtbased mass concentrations of Nafion films were different, suggesting that the deposited Nafion did not form a homogeneous rigid layer on the crystal surface (Fig. 2b). Moreover, the effects of the rinsing process on the properties of Nafion films were evaluated by quantifying the loss of IEC of the Nafion material as a function of the solution pH. Fig. S1 in the ESI $\dagger$ shows that the loss of IEC is not significant in the $\mathrm{pH}$ range of 2.0-8.0, implying that the rinsing process using ultrapure water $(\mathrm{pH}=6.50)$ should not change the acid sites of the Nafion film.

An interpretation of the $\Delta D / \Delta f$ from QCM-D results can provide insights into the viscoelastic properties of the adsorbed layers. As can be seen from Fig. 3a, $\left|\Delta D_{5} / \Delta f_{5}\right|$ values for all the three kinds of Nafion films are $>0.2 \times 10^{-6}$. Upon rinsing with

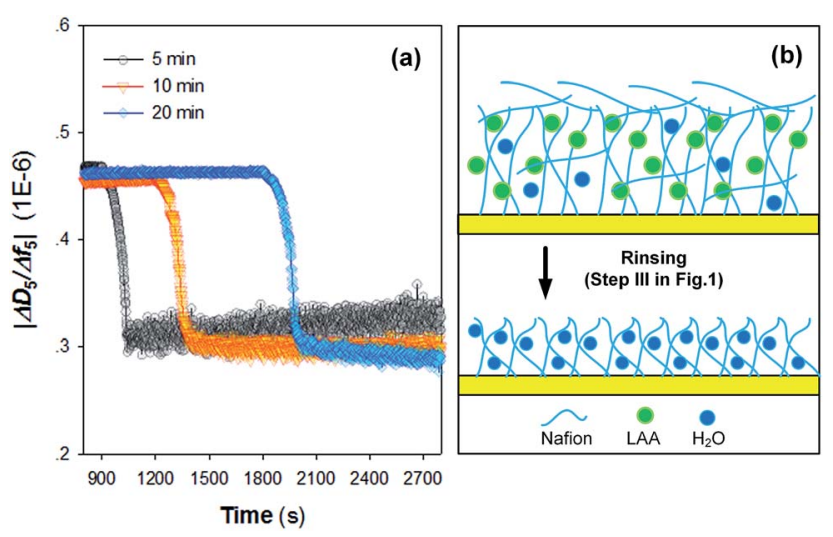

Fig. 3 (a) Time course results of $\left|\Delta D_{5} / \Delta f_{5}\right|$ changes during assembly of Nafion films on crystal sensors as a function of Nafion injection time; (b) schematic presentation of the structural transformation of the Nafion film upon rinsing with ultrapure water. LAA represents lower aliphatic alcohols. ultrapure water, $\left|\Delta D_{5} / \Delta f_{5}\right|$ values were all decreased. The large decrease in energy dissipation (Fig. 2a) is typical for a structural transformation from a dissipative non-rigid structure to a stiffer and most likely more compact structure, as observed in several earlier model systems. ${ }^{\mathbf{2 4 - 2 6}}$ It is generally expected that the assembly of heterogeneous Nafion film on the crystal sensors might begin with the interaction between the hydrophobic body parts of Nafion and crystal surface that leads to the formation of an adsorptive monolayer, ${ }^{27}$ followed by the further decoration of the undeformed Nafion resin as a result of the affinity and steric hindrance (Fig. 3b). After rinse, the loosely-adsorbed Nafion were likely removed from the film with partial leaching of the water-soluble LAA. ${ }^{28}$ As such, the layer became more rapid (lower $\left|\Delta D_{5} / \Delta f_{5}\right|$ values). ${ }^{29}$

Fig. 3 a suggests that the injection time also affects the variation of viscoelastic properties of the Nafion film, i.e., an injection time of Nafion solution over 10 min resulted in a more rigid film after rinsing. In view of the obtained results of this study, a Nafion injection time of 10 min was finally chosen for the assembly of Nafion film on the QCM-D crystal sensors.

\subsection{Adsorption behaviors of $\mathrm{Fe}(\mathrm{III})$ species}

Fig. 4 shows the adsorption and desorption behaviors of $\mathrm{Fe}(\mathrm{III})$ species on different surfaces. Two $\mathrm{pH}$ values (i.e., $\mathrm{pH}=2.37$ and 6.50) were evaluated in this study as they are typical for electrochemical systems operated in acidic and circumneutral conditions. It can be observed that the magnitude of frequency changes $\left(\Delta f_{5}\right)$ is highly associated with the nature of the sensor surfaces; i.e., after the injection of $10 \mathrm{mM} \mathrm{Fe(III)} \mathrm{for} 20 \mathrm{~min}$ (step IV in Fig. 1), $\Delta f_{5}$ were changed by -15.1 and $-20.1 \mathrm{~Hz}$ on the gold-plated crystal and Nafion film surface respectively, indicating the stronger interaction between Nafion and $\mathrm{Fe}$ (III) species. Specifically, a sharp increase in $\left|\Delta f_{5}\right|$ was noticed initially on the Nafion film (Fig. 4) followed by a process exhibiting pseudo-first-order growth with an apparent rate constant $\left(k_{\text {app }}\right)$ of $0.0017 \mathrm{~s}^{-1}$ (details could be found in Fig. S2 in

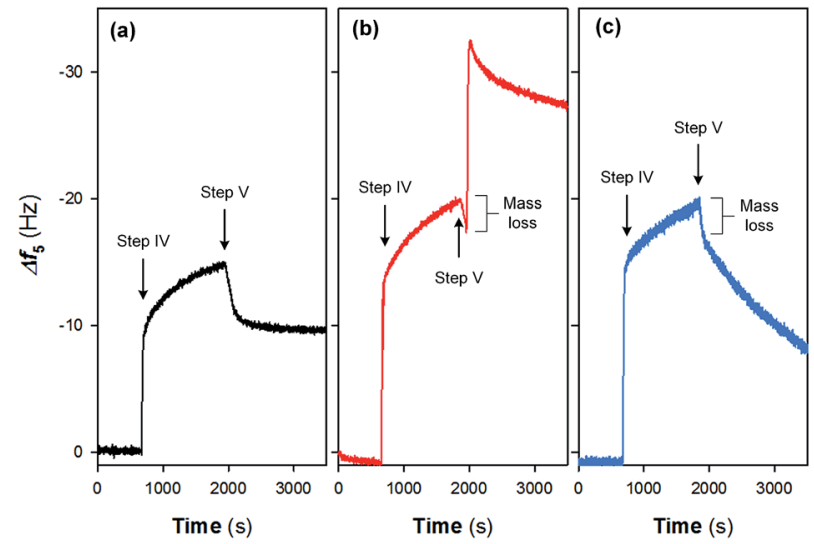

Fig. 4 Interfacial behaviors of $\mathrm{Fe}($ III) species on (a) pristine crystal surface followed by the rinse with ultrapure water $(\mathrm{pH}=6.50)$ and Nafion film surface followed by the rinse with (b) ultrapure water and (c) acid solution ( $\mathrm{pH}=2.37)$. Experimental conditions: injection time for step IV = 20 min; [Fe(॥)] = $10 \mathrm{mM}$. 
the ESI $\dagger$ ), attributed to the limited surface site number of Nafion and the constant Fe(III) concentration. Release behaviors of $\mathrm{Fe}(\mathrm{III})$ species from the pristine crystal surface and Nafion film were then evaluated at different $\mathrm{pH}$. When rinsed with neutral ultrapure water (Fig. $4 \mathrm{a}$ and b), $\left|\Delta f_{5}\right|$ of the Nafion film underwent a transient decrease followed by a notable jump and subsequent decline over time. In comparison, the jump of $\left|\Delta f_{5}\right|$ was not noticed during the rinsing process on the gold-plated crystal surface. Furthermore, it can be noted from Fig. 4c that continuous decline of $\left|\Delta f_{5}\right|$ occurred when the $\mathrm{Fe}(\mathrm{III})$ bound Nafion film subject to acidic elution ( $\mathrm{pH}$ of 2.37 ), with the observation of a rapid mass loss followed by a pseudo-first-order decay giving $k_{\text {app }}$ of $0.0007 \mathrm{~s}^{-1}$.

In environmental applications of the IEMs, although it is acknowledged that the interaction of $\mathrm{Fe}(\mathrm{III})$ species from the electrolyte with the functional groups (e.g., $\left.-\mathrm{SO}_{3} \mathrm{H}\right)$ leads to serious consequences such as the inhibition of ion transfer and increase in system resistance, ${ }^{7,8}$ synthesis of the adsorption behaviors of Fe(III) on IEM surfaces is still scarce. Based on the obtained results of this study and past literature, ${ }^{15,16}$ the following reaction pathways are therefore proposed (as shown schematically in Fig. 5):

Initially, a heterogeneous fouling layer is likely developed on the surface with exposure to water/wastewater containing Fe(III).

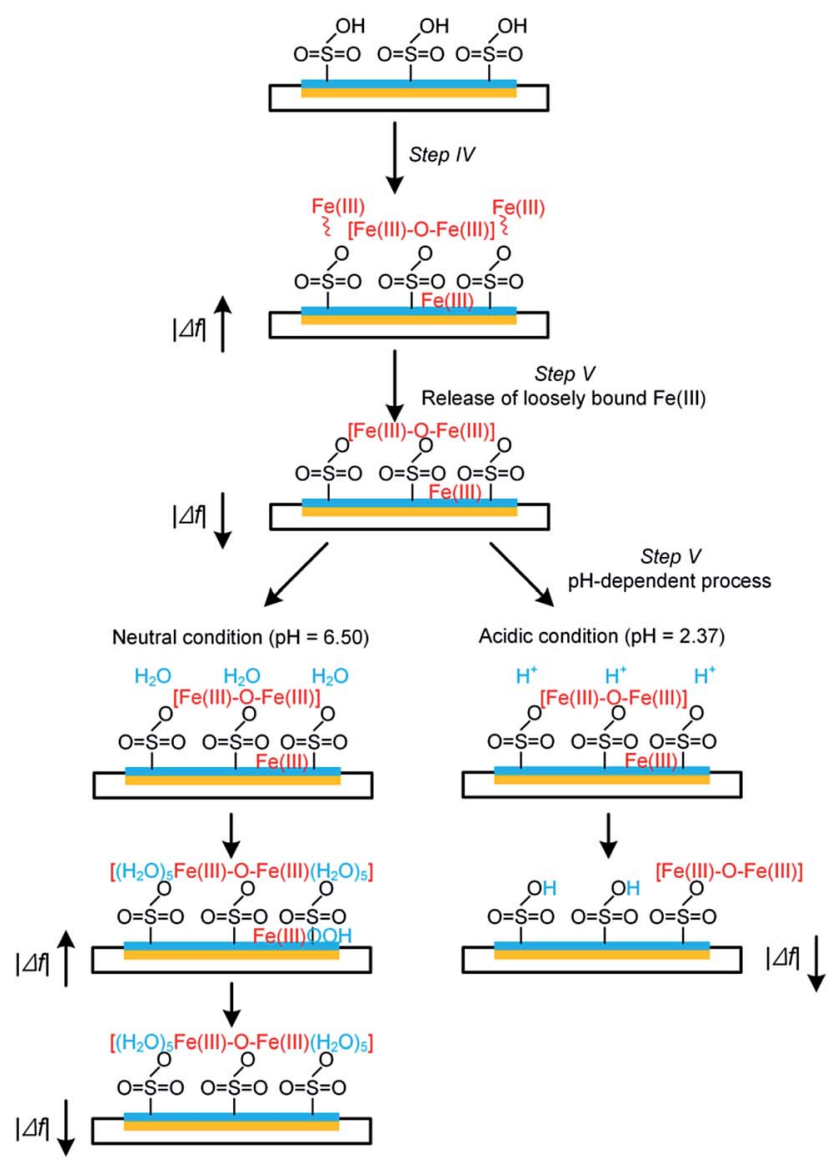

Fig. 5 A schematic presentation of Fe(III) interfacial behaviors on Nafion film surface at different $\mathrm{pH}$.
The formation of $\mathrm{Fe}(\mathrm{III})$ fouling can be associated with (i) the loosely binding of Fe(III) species on Nafion film surface, (ii) iron exchange of $\mathrm{Fe}(\mathrm{III})$ with $-\mathrm{SO}_{3} \mathrm{H}$ groups and/or (iii) trapping of $\mathrm{Fe}(\mathrm{III})$ species (e.g., Fe-(oxyhydr)oxides) in the cluster-channel. ${ }^{29}$ As can be seen from Fig. $4 \mathrm{~b}$ and $\mathrm{c}$, upon elution under either neutral or acidic condition, a rapid mass loss occurs at the initial stage of step V (Fig. 1), which suggests the ease of removing loosely bound Fe(III) species on the boundary of the fouling layer as a result of the back-diffusion. After this, release of Fe(III) exhibits a pH-dependent manner (Fig. 5): i.e., under the neutral condition, hydrolysis of the bound and/or trapped Fe(III) is generally expected due to the low solubility product of FeOOH. ${ }^{30}$ As such, a jump of $\left|\Delta f_{5}\right|$ is observed in Fig. 4 b. In contrast, $\mathrm{Fe}(\mathrm{III})$ species could readily be removed from the Nafion surface under the acidic condition. The lower $k_{\text {app }}$ $\left(0.0007 \mathrm{~s}^{-1}\right)$ for the release process compared to the $\mathrm{Fe}(\mathrm{III})$ adsorption process $\left(0.0017 \mathrm{~s}^{-1}\right)$ implied the strong interaction between $\mathrm{Fe}(\mathrm{III})$ and sulfonic groups. With prolonging of the rinsing time, decline of $\left|\Delta f_{5}\right|$ was also noted on the Nafion film under the neutral condition. Plausible explanations for this phenomenon might include (i) the leaching of the precipitates in the channel and/or (ii) the fretting of the Nafion film.

\subsection{Correlation of the QCM-D results with Nafion membrane performance}

The relationship of the QCM-D results with Nafion membrane performance was further evaluated by measuring the changes of functional groups and ohmic resistance of Nafion membranes as a result of Fe(III) deposition. Fig. 6a provides the ATR-FTIR spectra of pristine Nafion 117 film and the fouled one upon exposure to $10 \mathrm{mM} \mathrm{Fe(III)} \mathrm{solution} \mathrm{for} 60 \mathrm{~min}$. Although the two kinds of membranes have similar ATR-FTIR bands such as shoulder peaks at 970 and $982 \mathrm{~cm}^{-1}$ assigned to C-O-C linkages, symmetric $\mathrm{SO}_{3}{ }^{-}$stretching vibration at $1056 \mathrm{~cm}^{-1}$ and shoulder peaks at 1146 and $1203 \mathrm{~cm}^{-1}$ representing symmetric and asymmetric $\mathrm{CF}_{2}$ vibrations, ${ }^{\mathbf{3 1 , 3 2}}$ a major difference is the appearance of $\mathrm{Fe}-\mathrm{O}-\mathrm{Fe}$ antisymmetric stretching mode of the $\mu$ oxo bridged dimer at $870 \mathrm{~cm}^{-1}$ on the $\mathrm{Fe}(\mathrm{III})$ fouled membrane. ${ }^{15}$
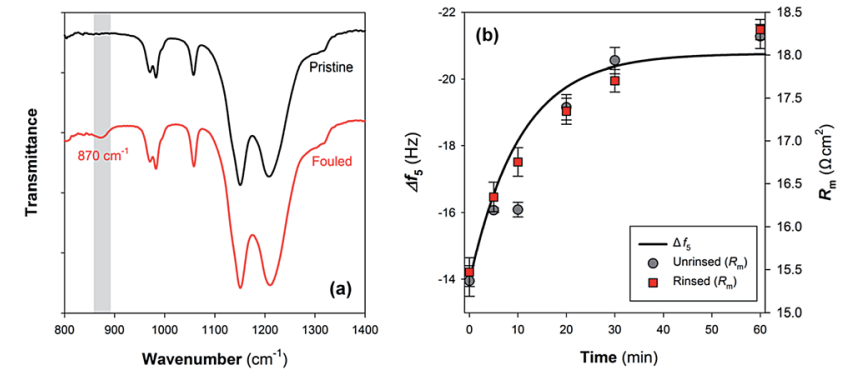

Fig. 6 (a) ATR-FTIR spectra of the pristine Nafion 117 film and the fouled one upon exposure to $10 \mathrm{mM} \mathrm{Fe}(\mathrm{III})$ solution for $60 \mathrm{~min}$. (b) Kinetics modeling of Fe(III) adsorption on the Nafion film and its prediction on membrane ohmic resistance $\left(R_{\mathrm{m}}\right)$. In $(\mathrm{b})$, the grey circles represent the $R_{\mathrm{m}}$ of Nafion membrane measured following the exposure to $10 \mathrm{mM}$ Fe(III) solution without rinse, and the red squares the $R_{\mathrm{m}}$ measured after the rinse with copious amount of ultrapure water ( $\mathrm{pH}$ of 6.50). 
Fig. S3 in the ESI $\dagger$ shows the changes in the membrane morphology following the binding of Fe(III) species. Small bumps were observed on the surface of the fouled Nafion 117 membrane with the average roughness increased.

As can be seen from Fig. 6b, the membrane ohmic resistance $\left(R_{\mathrm{m}}\right)$ is increased from $15.4 \pm 0.2 \Omega \mathrm{cm}^{2}$ to $18.2 \pm 0.1 \Omega \mathrm{cm}^{2}$ upon fouling in $10 \mathrm{mM} \mathrm{Fe(III)} \mathrm{solution} \mathrm{for} 60 \mathrm{~min}$. Considering that the initially rapid jump of $\left|\Delta f_{5}\right|$ that might be associated with (i) the change of the properties of the fluid and/or (ii) loosely binding of Fe(III) species on Nafion film surface did not cause a transient variation of ohmic resistance (Fig. $4 \mathrm{~b}$ and $6 \mathrm{~b}$ ), part of $\left|\Delta f_{5}\right|$ exhibiting a pseudo-first-order growth (Fig. S2 in the ESI $\dagger$ ) was then used to predict the time course results of $R_{\mathrm{m}}$. Fig. $6 \mathrm{~b}$ shows that the interaction between aqueous Fe(III) and Nafion surface functional groups should be responsible for the deterioration of membrane performance. A similar conclusion could be drawn in view of the change of specific resistance $\left(\rho_{\mathrm{m}}\right)$ of the membranes (Fig. S4 in the ESI $\dagger$ ). The QCM-D results can provide a reasonable interpretation for the temporal change of $R_{\mathrm{m}}$.

Furthermore, it has been reported that $\mathrm{Fe}(\mathrm{III})$ precipitation due to $\mathrm{pH}$ variation is an important factor contributing to inorganic and complex fouling in membrane filtration processes. $^{33,34}$ While hydrolysis of $\mathrm{Fe}(\mathrm{III})$ and/or structural transformation of $\mu$-oxo bridged dimer to edge-sharing $\mathrm{FeO}_{6}$ octahedra are generally expected when providing neutral elution (Fig. 4b and 5), this seems not to cause a further increase of $R_{\mathrm{m}}$ : i.e., there is no significant difference between the measured results using unrinsed and rinsed samples (Fig. 6b). As such, we can preliminarily conclude that on condition that Nafion surface sites are occupied by multivalent metal ions, the membrane conductivity might not be lowered due to the structural transformation of these species, despite the reservation remaining that crystallization of $\mathrm{Fe}(\mathrm{III})$ can cause changes of membrane surface physicochemical properties (e.g., roughness) resulting in possible interaction with other foulants and deterioration of membrane performance., ${ }^{9,35}$

In the present work, we present a novel approach to analyze the chemical scaling on IEMs. Membrane thin films can be formed on the crystal surfaces via sedimentation and/or spincoating methods, ${ }^{36}$ with the potentially expanded applications in electrochemical membrane process including (i) the measurement of molecular adsorption and/or interactions taking place on the surface (ii) development and evaluation of novel, antifouling conductive materials. Compared to the ex situ characterizing technologies (e.g., SEM and ATR-FTIR), QCM-D could provide a real-time analysis of the interfacial behavior of ions and biopolymers on the IEM surface with the interpretation of $\Delta D$ and $\Delta f$ revealing the viscoelastic properties of the fouling layers. ${ }^{25,37}$ Consideration could also be given to the effects of different environmental parameters (e.g., temperature and ionic strength) on the fouling behavior and performance of IEMs under real conditions. Moreover, of particular interest is a recent advance of Q-Sense module, QEM 401 that enables simultaneous QCM-D and electrochemical impedance spectroscopy measurements. ${ }^{37}$ There is an illumination that QCM-D experiments can provide real-time information on structure and interfacial charge transfer of thin films in the presence of electric field, from which important rate coefficients can be retrieved and used for (i) the modelling and/or (ii) prediction of the transport of concerned foulants on conductive membranes in future research.

\section{Conclusions}

In this study, Nafion thin film was successfully formed on the crystal surface using a sedimentation method. The Nafion film exhibited a non-rigid structure after the rinse, with a Voigtbased mass concentration of $\sim 500 \mathrm{ng} \mathrm{cm}^{-2}$ at a resin injection time of Nafion solution for $10 \mathrm{~min}$. The QCM-D results evidenced the adsorption behaviors of Fe(III) on Nafion film surface: i.e., a transient deposition of Fe(III) likely occurred at the initial stage, followed by a pseudo-first-order ongoing process probably as a result of the interaction between $\mathrm{Fe}(\mathrm{III})$ and Nafion surface sites that retarded the proton transfer consequently. Although the variation pattern of $\Delta f_{5}$ upon neutral elution indicated the hydrolysis of Fe(III) and/or structural transformation of $\mu$-oxo bridged dimer, the electrochemical measurements indicated that this did not cause a further deterioration of Nafion performance.

\section{Conflicts of interest}

There are no conflicts of interest to declare.

\section{Acknowledgements}

This work is financially supported by National Natural Science Foundation of China (51378371 \& 51422811) of China. Dr Jinxing $\mathrm{Ma}$ is a recipient of the Vice-Chancellor's Postdoctoral Research Fellow of UNSW Australia (RG152482).

\section{References}

1 M. A. Hickner, H. Ghassemi, Y. S. Kim, B. R. Einsla and J. E. McGrath, Chem. Rev., 2004, 104, 4587-4611.

2 X. Cao, X. Huang, P. Liang, K. Xiao, Y. Zhou, X. Zhang and B. E. Logan, Environ. Sci. Technol., 2009, 43, 7148-7152.

3 S. Porada, R. Zhao, A. Van Der Wal, V. Presser and P. Biesheuvel, Prog. Mater Sci., 2013, 58, 1388-1442.

4 S. Porada, D. Weingarth, H. V. M. Hamelers, M. Bryjak, V. Presser and P. M. Biesheuvel, J. Mater. Chem. A, 2014, 2, 9313-9321.

5 Z. Wang, J. Ma, Y. Xu, H. Yu and Z. Wu, J. Power Sources, 2013, 235, 280-288.

6 J. Ma, D. He, W. Tang, P. Kovalsky, C. He, C. Zhang and T. D. Waite, Environ. Sci. Technol., 2016, 50, 13495-13501.

7 J. Xu, G.-P. Sheng, H.-W. Luo, W.-W. Li, L.-F. Wang and H.-Q. Yu, Water Res., 2012, 46, 1817-1824.

8 M.-J. Choi, K.-J. Chae, F. F. Ajayi, K.-Y. Kim, H.-W. Yu, C.-w. Kim and I. S. Kim, Bioresour. Technol., 2011, 102, 298-303.

9 J. Ma, Z. Wang, D. Suor, S. Liu, J. Li and Z. Wu, J. Power Sources, 2014, 272, 24-33. 
10 I. A. Stenina, P. Sistat, A. I. Rebrov, G. Pourcelly and A. B. Yaroslavtsev, Desalin. Water Treat., 2004, 170, 49-57.

11 F. Harnisch, U. Schröder and F. Scholz, Environ. Sci. Technol., 2008, 42, 1740-1746.

12 T. Okada, Y. Ayato, M. Yuasa and I. Sekine, J. Phys. Chem. B, 1999, 103, 3315-3322.

13 M. Ben Sik Ali, D. Jellouli Ennigrou and B. Hamrouni, Environ. Technol., 2013, 34, 2521-2529.

14 A. ter Heijne, H. V. M. Hamelers, V. de Wilde, R. A. Rozendal and C. J. N. Buisman, Environ. Sci. Technol., 2006, 40, 52005205.

15 M. Yamaguchi and A. Ohira, Comput. Theor. Chem., 2015, 1071, 61-67.

16 H. K. Pan, A. Meagher, M. Pineri, G. S. Knapp and S. L. Cooper, J. Chem. Phys., 1985, 82, 1529-1538.

17 A. Sweity, W. Ying, S. Belfer, G. Oron and M. Herzberg, J. Membr. Sci., 2011, 378, 186-193.

18 M. Hashino, K. Hirami, T. Ishigami, Y. Ohmukai, T. Maruyama, N. Kubota and H. Matsuyama, J. Membr. Sci., 2011, 384, 157-165.

19 Z. Wang, F. Huang, X. Mei, Q. Wang, H. Song, C. Zhu and Z. Wu, J. Membr. Sci., 2014, 471, 258-264.

20 K. D. Kwon, H. Green, P. Bjöörn and J. D. Kubicki, Environ. Sci. Technol., 2006, 40, 7739-7744.

21 P. Yi and K. L. Chen, Environ. Sci. Technol., 2013, 47, 1221112218.

22 H. Chen, J. D. Snyder and Y. A. Elabd, Macromolecules, 2008, 41, 128-135.

23 D. R. Morris and X. Sun, J. Appl. Polym. Sci., 1993, 50, 14451452.
24 F. Hook, B. Kasemo, T. Nylander, C. Fant, K. Sott and H. Elwing, Anal. Chem., 2001, 73, 5796-5804.

25 A. E. Contreras, Z. Steiner, J. Miao, R. Kasher and Q. L. Li, Environ. Sci. Technol., 2011, 45, 6309-6315.

26 O. Furman, S. Usenko and B. L. T. Lau, Environ. Sci. Technol., 2013, 47, 1349-1356.

27 G. C. Abuin, M. Cecilia Fuertes and H. R. Corti, J. Membr. Sci., 2013, 428, 507-515.

28 G. Raj, C. Swalus, M. Delcroix, M. Devillers, C. DupontGillain and E. M. Gaigneaux, J. Colloid Interface Sci., 2015, 445, 24-30.

29 K. A. Mauritz and R. B. Moore, Chem. Rev., 2004, 104, 45354585.

30 A. N. Pham, A. L. Rose, A. J. Feitz and T. D. Waite, Geochim. Cosmochim. Acta, 2006, 70, 640-650.

31 M. Danilczuk, L. Lin, S. Schlick, S. J. Hamrock and M. S. Schaberg, J. Power Sources, 2011, 196, 8216-8224.

32 Z. Liang, W. Chen, J. Liu, S. Wang, Z. Zhou, W. Li, G. Sun and Q. Xin, J. Membr. Sci., 2004, 233, 39-44.

33 Z. Wang, J. Ma, C. Y. Tang, K. Kimura, Q. Wang and X. Han, J. Membr. Sci., 2014, 468, 276-307.

34 P. Le-Clech, V. Chen and T. A. Fane, J. Membr. Sci., 2006, 284, 17-53.

35 F. Meng, B. Liao, S. Liang, F. Yang, H. Zhang and L. Song, J. Membr. Sci., 2010, 361, 1-14.

36 S. Iwamori, K. Yoshino, H. Matsumoto, K. Noda and I. Nishiyama, Sens. Actuators, B, 2012, 171-172, 769-776.

37 E. Briand, M. Zäch, S. Svedhem, B. Kasemo and S. Petronis, Analyst, 2010, 135, 343-350. 